\title{
The $1 / N$-Expansion as a Perturbation about the Mean Field Theory: A One-Dimensional Fermi Model
}

\author{
D.H.U. Marchetti ${ }^{1, \star}$, P.A. Faria da Veiga ${ }^{2, \star \star}$, T.R. Hurd ${ }^{3, \star \star \star}$ \\ ${ }^{1}$ Instituto de Física, Universidade de São Paulo, 01452 São Paulo, SP, Brazil \\ 2 ICMSC, Universidade de São Paulo, 13560 São Carlos, SP, Brazil \\ 3 Department of Mathematics \& Statistics, McMaster University, Hamilton, Canada L8S 4K1
}

Received: 5 April 1995/Accepted: 15 January 1996

\begin{abstract}
We examine a family of probability measures on $\mathbf{R}^{L}$ with real parameter $\zeta>0$ and integer parameters $N, L>0$. Each such measure is equivalent to the lattice version of a one-dimensional discrete chiral-invariant fermionic quantum field theory with quartic interaction, with $N$ the number of flavours. After applying the Matthews-Salam formula, the model becomes a statistical mechanical model of a chain of continuous Gaussian spins, coupled with a certain non-standard weight function. Finally, the model can also be considered as a probability measure on the set of tridiagonal matrices with fixed off-diagonal and random diagonal entries.

Our analysis shows how to develop an asymptotic expansion in $1 / N$, valid for all $L$ and $\zeta$, for the fundamental expectation values. From this it follows that the two point fermion correlation function decays with a mass which agrees to the leading order in $1 / N$ with the mean field value calculated by the argument of Gross-Neveu. The analytical technique we develop in essence combines a transfer matrix method with the Laplace method (steepest descent) for asymptotics of integrals.
\end{abstract}

\section{Introduction}

It was argued some years ago [NJ, GN] that a chiral invariant fermion theory with quartic interaction will acquire a mass dynamically by spontaneous symmetry breaking. The effective potential shows degenerate minima in the one-loop approximation, leading to a ground state with non-vanishing expectation of $\bar{\psi} \psi$.

Rigorous control of multi-phase theories was developed by Glimm-Jaffe-Spencer [GJS] who devised an expansion about the mean field theory by combining a cluster expansion with an expansion in phase boundaries.

The present paper is part of a program which aims at understanding the mechanism by which mass is generated dynamically in models with four-fermion interactions. We here consider a one-dimensional discrete chiral lattice model and show

\footnotetext{
$\star$ Partially supported by FAPESP and CNPq.

$\star \star$ Partially supported by CNPq.

$\star \star \star$ Supported by the Natural Sciences and Engineering Research Council of Canada.
} 
that a fermion mass is generated without symmetry breaking. Our procedure combines transfer matrices with a steepest descent method to produce an asymptotic expansion in $1 / N$ which agrees to leading order with the mean field theory and can be viewed as an alternative to the expansion proposed in reference [GJS].

1.1. The Model. For fixed $\zeta>0$ and integers $N>0, L$ even, we consider the joint probability measure $\mu_{L}(\phi)$ on $\mathbf{R}^{L}$, whose density $g_{L}(\phi)$ is given by

$$
g_{L}(\phi)=\frac{1}{Z_{L}} \frac{e^{-\frac{1}{2}(\phi, \phi)}}{(2 \pi)^{L / 2}} \operatorname{det}\left(\Gamma_{L}+(\zeta / \sqrt{N}) \Phi_{L}\right)^{2 N},
$$

where $(\phi, \phi)=\sum_{i=1}^{L} \phi_{i}^{2}$ and

$\Gamma_{L}=\left(\begin{array}{ccccc}0 & 1 & 0 & \cdots & 0 \\ -1 & 0 & 1 & \cdots & 0 \\ 0 & -1 & 0 & \cdots & 0 \\ \vdots & \vdots & \vdots & \ddots & \vdots \\ 0 & 0 & 0 & \cdots & 0\end{array}\right)$ and $\Phi_{L}=\left(\begin{array}{ccccc}\phi_{1} & 0 & 0 & \cdots & 0 \\ 0 & \phi_{2} & 0 & \cdots & 0 \\ 0 & 0 & \phi_{3} & \cdots & 0 \\ \vdots & \vdots & \vdots & \ddots & \vdots \\ 0 & 0 & 0 & \cdots & \phi_{L}\end{array}\right)$

are $L \times L$ matrices. The partition function

$$
Z_{L}=\int \prod_{i=1}^{L}\left(\frac{d \phi_{i}}{\sqrt{2 \pi}}\right) e^{-\frac{1}{2}(\phi, \phi)} \operatorname{det}\left(\Gamma_{L}+(\zeta / \sqrt{N}) \Phi_{L}\right)^{2 N}
$$

normalizes the measure.

We will be interested in the large $N$ behavior of the limiting measure $\mu$ obtained from $\mu_{L}$ as $L \rightarrow \infty$. The existence of this limit will be a consequence of our analysis.

The measure $\mu_{L}$ arises from the application of the Matthews-Salam formula to the one dimensional fermionic system on a lattice $\Lambda \subset a \mathbf{Z}$ with $|\Lambda|=L$, described by the action

$$
\mathscr{A}(\bar{\psi}, \psi)=a\left\{\sum_{x \in \Lambda} \bar{\psi}_{x} \cdot(i \mathbf{D} \psi)_{x}+\frac{\lambda^{2}}{2 N} \sum_{x \in \Lambda}\left(\bar{\psi}_{x} \cdot \psi_{x}\right)^{2}\right\} .
$$

Here $\mathscr{A}(\bar{\psi}, \psi)$ is a functional on the Grassmann algebra generated by the fermionic field $(\bar{\psi}, \psi)$ with scalar product, $\bar{\psi} \cdot \chi=\sum_{j, \alpha} \bar{\psi}_{\alpha}^{j} \chi_{\alpha}^{j}$, running over "flavours" $j=1, \ldots, N$, and spins, $\alpha=\uparrow, \downarrow$. $i \mathbf{D}$ is the symmetric difference operator whose kernel is given by

$$
(i \mathbf{D})_{x y}=\frac{1}{a}\left(\Gamma_{L}\right)_{\frac{x}{a} \frac{y}{a}} \sigma_{3},
$$

where $a$ is the lattice spacing and $\sigma_{3}$ the diagonal Pauli matrix. $a \lambda^{2}=\zeta^{2}$ is a dimensionless coupling constant.

This equivalence requires in particular the elimination of the spin degrees of freedom. To see how this can happen, we set $\boldsymbol{\Phi}=\Phi_{L} \otimes 1_{2}$, where $1_{n}$ is the $n \times n$ identity and notice that, due to the fact $\Gamma_{L}^{T}=-\Gamma_{L}$,

$$
\operatorname{det}(i a \mathbf{D}+(\zeta / \sqrt{N}) \boldsymbol{\Phi})^{N}=\operatorname{det}\left(\Gamma_{L}+(\zeta / \sqrt{N}) \Phi_{L}\right)^{2 N} .
$$

This (det) $)^{N}$ can now be written as the $N$-flavour Grassmann integral

$$
\int \prod_{x \in \Lambda} d \bar{\psi}_{x} d \psi_{x} \exp \left\{\sum_{x \in \Lambda} \bar{\psi}_{x} \cdot[(i a \mathbf{D}+(\zeta / \sqrt{N}) \boldsymbol{\Phi}) \psi]_{x}\right\} \text {. }
$$


The Matthews-Salam formula is based on the identity

$$
e^{\left(\zeta^{2} / 2 N\right)(\bar{\psi} \cdot \psi)^{2}}=\frac{1}{\sqrt{2 \pi}} \int d \phi e^{-\frac{1}{2} \phi^{2}} e^{(\zeta / \sqrt{N})(\bar{\psi} \cdot \psi) \phi} .
$$

Using (1.5)-(1.7), the partition function (1.3) can thus be written as a Grassmann integral

$$
Z_{L}=\int \prod_{x \in \Lambda} d \bar{\psi}_{x} d \psi_{x} e^{\mathscr{A}(\bar{\psi}, \psi)}
$$

\section{Remarks.}

1. The action (1.4) is the one-dimensional Euclidean lattice version (the only direction is time) of the Gross-Neveu model [GN]. The equivalence between (1.3) and (1.8) can be easily extended to $d$-dimensional regular lattices with $d>1$, Cayley trees and other graphs [FHM]: If $\mathscr{G}$ is a graph with $L$ sites labeled by $\{1, \ldots, L\}$, $\Phi_{L}$ is as in (1.2) and $\Gamma_{L}$ is a conveniently defined skew-symmetric matrix with $\left(\Gamma_{L}\right)_{i j}= \pm 1$ if $i, j$ are linked sites in $\mathscr{G}$ and 0 otherwise.

2. The model in $a \mathbf{Z}^{2}$ is of special interest since the massive version of (1.4) is perturbatively renormalizable and asymptotically free in the ultraviolet [GK, FMRS] (provided $N>1$ ), which allows one to remove the lattice cutoff by a limiting procedure. Our results in $d=1$ are uniform in the lattice spacing $a$ provided $\lambda=\lambda(a)=\zeta / \sqrt{a}$ for any $0<\zeta<\infty$.

3 . The symmetry $\phi \rightarrow-\phi$ of the measure (1.1) corresponds to the discrete chiral symmetry

$$
\psi \rightarrow e^{i \frac{\pi}{2} \sigma_{2}} \psi, \quad \bar{\psi} \rightarrow \bar{\psi} e^{i \frac{\pi}{2} \sigma_{2}},
$$

of the action (1.4).

4. Most of our results are valid if we consider a measure $v_{L}$ defined in $\mathbf{C}^{L}$ whose density is given by (1.1) with $\operatorname{det}(\Gamma+(\zeta / \sqrt{N}) \Phi)$ replaced by $|\operatorname{det}(\Gamma+(\zeta / \sqrt{N}) \Phi)|$. By the Matthews-Salam formula, this measure represents a fermion system described by the action (1.4) with the local interaction $\left(\bar{\psi}_{x} \cdot \psi_{x}\right)^{2}$ replaced by

$$
\left(\bar{\psi}_{x} \cdot \psi_{x}\right)^{2}-\left(\bar{\psi}_{x} \cdot \sigma_{2} \psi_{x}\right)^{2}
$$

where $\sigma_{2}$ is the imaginary Pauli matrix. Notice that (1.9) is invariant under the continuous chiral transformation

$$
\psi \rightarrow e^{i \frac{\theta}{2} \sigma_{2}} \psi, \quad \bar{\psi} \rightarrow \bar{\psi} e^{i \frac{\theta}{2} \sigma_{2}}, \quad \theta \in[0,2 \pi) .
$$

The measure $v_{L}$ is thus $U(1)$ invariant.

1.2. The Mean Field Approximation and Peierls Estimate. As usual, the first step in understanding a probability measure in equilibrium statistical mechanics is to consider the mean field approximation. It consists here in conditioning the measure $\mu_{L}(\phi)$ on constant configurations $\left\{\phi_{i}=\varphi\right\}_{i=1}^{L}$. Denoting the mean field density by $h_{L}(\varphi)$, it follows that:

Proposition 1.1. For any $\zeta>0$ and $L>2 / \zeta^{2}-2$,

(i) $h_{L}(\varphi)=h_{L}(-\varphi)$, 
(ii) $h_{L}(\varphi)$ achieves its maximum at $\varphi= \pm \varphi^{*} \equiv \pm m_{L} \sqrt{N} / \zeta$, where $m_{L}=m_{L}(\zeta)$ is the unique positive solution of the "mean-field" equation

$$
m=2 \zeta^{2} \frac{1}{L} \frac{f_{L}^{\prime}(m)}{f_{L}(m)}
$$

where $f_{L}(m)=\operatorname{det}\left(\Gamma_{L}+m 1_{L}\right)$ (note that (1.10) does not depend on $\left.N\right)$.

(iii) For large $L, m_{L}$ is given by

$$
m_{L}=m(\zeta)+O(1 / L)
$$

where $m(\zeta)=\left(2\left(1+\zeta^{4}\right)^{1 / 2}-2\right)^{1 / 2}$. Furthermore, $\varphi=0$ is a local minimum of $h_{L}(\varphi)$ and

$$
\frac{h_{L}\left( \pm \varphi^{*}\right)}{h_{L}(0)} \geqq e^{c L N}
$$

for some constant $c=c(\zeta)>0$.

From Proposition 1.1, the effective potential (see details in Sect. 3)

$$
V_{L}(\varphi)=\frac{-1}{L} \ln h_{L}(\varphi)
$$

is a symmetric double-well-shaped potential with minima separated by a barrier of width $O(\sqrt{N})$ and height $O(N)$ at $\varphi=0$. When $N \rightarrow \infty$, the density $h_{L}(\varphi)$ can be well approximated by a sum of two Gaussians centred at $\varphi= \pm \varphi^{*}$, corresponding to two pure phases. The expectation with respect to the mean field measure of any function $K(\varphi)$ is of the form of a Laplace integral. It is thus given, in the limit as $L \rightarrow \infty$, by

$$
\mathbf{E}_{h}[K] \equiv \lim _{L \rightarrow \infty} \int_{-\infty}^{\infty} d \varphi h_{L}(\varphi) K(\varphi)=\frac{1}{2}\left(K\left(\varphi^{*}\right)+K\left(-\varphi^{*}\right)\right) .
$$

To refine this double-well picture, it is necessary to have control over large fluctuations between the two minima (i.e. a Peierls estimate) and small fluctuations about the minima. This is usually done by use of an explicit $-\int(\nabla \phi)^{2}$ term in the action. In our present problem, no such gradient term is present. As an indication that nonetheless the measure does inhibit large fluctuations, we have a Peierls-type estimate:

Proposition 1.2 (Peierls Estimate). Given an Ising-like configuration $\left\{\sigma_{i}= \pm m\right\}$, $i=1, \ldots, L$, let us denote by $\sigma_{L}$ the diagonal matrix whose entries are $\sigma_{i} \delta_{i j}$ and set $f_{L}(\sigma)=\operatorname{det}\left(\Gamma_{L}+\sigma_{L}\right)$. There is $m_{0}$ such that for $m>m_{0}$ there exists $\delta<1$, uniformly in L, such that

$$
\left|\frac{f_{L}(\sigma)}{f_{L}(m)}\right| \leqq \delta^{p},
$$

where $p$ is the number of flips in the spin configuration $\sigma_{L}$.

In higher dimensions, such an estimate is an indicator of symmetry breaking: at low temperatures, this energy estimate dominates over the entropy estimate, and the system shows multiple phases. In one-dimension as here, no such conclusion is possible, since the entropy estimate dominates at all temperatures (i.e. all values of $\zeta$ ).

Mass generation is in fact expected to occur in the two or higher dimensional lattice Gross-Neveu model (1.4). Bałaban and Gawedzki [BG], for example, have 
established spontaneous symmetry breaking in the two-dimensional pseudoscalar Yukawa model, where an explicit gradient term is present. Recently mass generation in the two-dimensional Gross-Neveu model at large $N$ has been proved by Kopper, Magnen and Rivasseau [KMR] with a different choice of ultraviolet cutoff than the lattice cutoff we use.

Before we turn to the original measure (1.1), we shall write the fermion mass gap value in the mean field approximation. For this, we introduce the two-point function

$$
\left\langle\bar{\psi}_{x} \cdot \psi_{y}\right\rangle_{L}=\frac{1}{Z_{L}} \int \prod_{x \in \Lambda} d \bar{\psi}_{x} d \psi_{x} e^{\mathscr{A}(\bar{\psi}, \psi)} \bar{\psi}_{x} \cdot \psi_{y}
$$

and denote by $\left\langle\bar{\psi}_{x} \cdot \psi_{y}\right\rangle$ its thermodynamic limit. The fermion mass gap $\hat{m}$ (inverse correlation length) is then defined by

$$
\hat{m}=\lim _{|x-y| \rightarrow \infty} \frac{-1}{|x-y|} \ln \left|\left\langle\bar{\psi}_{x} \cdot \psi_{y}\right\rangle\right| .
$$

The mean field mass gap $\hat{m}_{M F}$ is obtained by setting $\phi_{i}=\varphi, i=1, \ldots, L$, in the Matthews-Salam representation of $\left\langle\bar{\psi}_{x} \cdot \psi_{x}\right\rangle$.

Proposition 1.3. The mean field mass gap $\hat{m}_{M F}$, is given by

$$
\hat{m}_{M F}=\frac{1}{2 a} \ln \left[\zeta^{2}+\left(1+\zeta^{4}\right)^{1 / 2}\right]
$$

1.3. The Main Results. Our first main result controls the partition function of the model.

Theorem 1.4. Given $\zeta \in(0, \infty)$, there exist $N_{0}=N_{0}(\zeta), c_{0}=c_{0}(\zeta)>0$ and $c_{1}=c_{1}(N, \zeta) \in(0,1)$, such that, if $N>N_{0}$ the following holds:

$$
Z_{L}=c_{1}\left[c_{0} e^{v N}(1+O(1 / N))\right]^{L}\left[1+O\left(\left(\mu_{1}^{(0)}\right)^{L}\right)\right] .
$$

Here $v>0$ is given by

$$
v=\sup _{(x, y) \in \mathscr{I}} f(x, y)-\ln \frac{e}{2 \zeta^{2}}>0,
$$

where $\mathscr{I}=\left\{\left(z, z^{\prime}\right) \in[0,1]^{2}: z+z^{\prime} \leqq 1\right\}$,

$$
f(x, y)=g(x)+g(y)-(1-x-y) \ln (1-x-y)
$$

with

$$
g(z)=(1-z) \ln (1-z)-z\left(\ln z-\ln \frac{e}{2 \zeta^{2}}\right)
$$

and $\mu_{1}^{(0)}=\mu_{1}^{(0)}(\zeta)<1$ is an eigenvalue of an associated integral equation (4.16).

Remark. $f(x, y)$ is a concave symmetric function with maximum at $(\bar{x}, \bar{x}) \in \mathscr{I}$, where

$$
\bar{x}=\frac{1}{2}\left(1+\frac{1}{\zeta^{2}}\right)-\frac{1}{2}\left(1+\frac{1}{\zeta^{4}}\right)^{\frac{1}{2}}
$$

varies from 0 to $\frac{1}{2}$ as $\zeta$ varies from $\infty$ to 0 . 
The positivity of $v$ leads to a free energy

$$
F=\lim _{L \rightarrow \infty}(1 / L) \ln Z_{L}=v N+O(1) .
$$

The next result shows that the fermion two point function has an exponential decay rate given to zeroth order in $1 / N$ by the mean field value:

Theorem 1.5. For any $\zeta \in(0, \infty)$, there exists $N_{0}=N_{0}(\zeta)$, such that for $N>N_{0}$ the following upper and lower bounds:

$$
C_{1} e^{-\hat{m}|x-y|} \leqq\left\langle\bar{\psi}_{x} \cdot \psi_{y}\right\rangle \leqq C_{2} e^{-\hat{m}|x-y|}
$$

hold with $\hat{m}=\hat{m}(\zeta)=\hat{m}_{M F}+O(1 / N)$.

The proof of Theorems 1.4 and 1.5 is based on a formula which expresses $\operatorname{det}\left(\Gamma_{L}+(\zeta / \sqrt{N}) \Phi_{L}\right)$ as the generating function of a monomer-dimer problem (see e.g. [L]) with the monomer activity at site $i$ proportional to the random field $\phi_{i}$ (Theorem 2.3). This provides us with a way to write the partition function $Z_{L}$ as the trace of a $(N+1) \times(N+1)$ symmetric matrix $T$ raised to the power $L$, multiplied by a projection matrix to enforce the correct boundary condition at each end. For small $N, T$ can be explicitly diagonalized with its largest eigenvalue governing the behavior of expectation values in the measure $\mu_{L}$. When $N$ is large, $T$ is approximated by an integral transfer matrix whose eigenvalues can be evaluated by the Laplace method.

The main results of this paper, Theorems 1.4 and 1.5, give the sense in which the $1 / N$-expansion is an expansion about the mean field theory.

This paper is organized as follows. In Sect. 2 we present our determinant expansion. We prove Propositions 1.1, 1.2 and 1.3 in Sect. 3. Theorem 1.4 will be proven in Sect. 4. Section 5 is a general discussion of higher order terms in the $1 / N$ expansion. The generation of a mass gap will be analyzed in Sect. 6. Finally, in the concluding section, we discuss features of the model which concern correlations of the $\phi$ field (i.e. $\bar{\psi} \psi$ correlations).

\section{The Monomer-Dimer Expansion}

We start by writing the determinant in (1.1) in a power series of $\left\{\phi_{j}\right\}_{j=1}^{L}$. Let $\mathscr{L}=[1, L] \cap \mathbf{Z}, L$ even, and set

$$
F_{L}(\vartheta)=\operatorname{det}\left(\Gamma_{L}+\vartheta_{L}\right)
$$

(later we shall set $\left.\vartheta_{i}=(\zeta / \sqrt{N}) \phi_{i}, i=1, \ldots, L\right)$. Since the determinant is a multilinear function of the matrix elements, the Taylor expansion of $F_{L}$ is given by

$$
F_{L}(\vartheta)=\sum_{Y \subseteq \mathscr{L}} \frac{\partial F_{L}}{\partial \vartheta_{Y}}(0) \vartheta_{Y}
$$

where $\partial / \partial \vartheta_{Y}$ is shorthand for the multiple derivative $\prod_{i \in Y} \partial / \partial \vartheta_{i}$ and $\vartheta_{Y}=\prod_{i \in Y} \vartheta_{i}$.

To write $F_{L}$ as a monomer-dimer problem, we introduce some notation. For a given $X \subseteq \mathscr{L}, \bar{X}$ denotes the subset of $[-1 / 2, L+1 / 2]$ obtained by replacing each $i \in X$ by the unit interval centred at site $i$. Let $\left(\bar{X}_{1}, \ldots, \bar{X}_{n}\right)$ be the decomposition of $\bar{X}$ into disjoint subsets and let $\left(X_{1}, \ldots, X_{n}\right)$ be the partition of $X$ with $X_{i}=\bar{X}_{i} \cap \mathbf{Z}$. Calling this the regular partition of $X$, it is such that all sites in $X_{i}$ are in consecutive order and $\operatorname{dist}\left(X_{i}, X_{j}\right) \geqq 2, i \neq j$. 
Lemma 2.1. For $X \subseteq \mathscr{L}$, let $\left(X_{1}, \ldots, X_{n}\right)$ be the regular partition of $X$. If $Y=\mathscr{L} \backslash X \equiv X^{c}$, then

$$
\frac{\partial F_{L}}{\partial \vartheta_{Y}}(0)=\operatorname{det} \Gamma_{\left|X_{1}\right|} \operatorname{det} \Gamma_{\left|X_{2}\right|} \cdots \operatorname{det} \Gamma_{\left|X_{n}\right|}
$$

is equal to 1 provided $\left|X_{i}\right|$ is even for all $i=1, \ldots, n$ and 0 otherwise.

Proof. To prove (2.3) it suffices to show that

$$
\frac{\partial F_{L}}{\partial \vartheta_{i}}(\vartheta)=F_{i-1}\left(\vartheta_{\{<i\}}\right) F_{L-i}\left(\vartheta_{\{>i\}}\right) .
$$

This follows by noting that $\partial F_{L} / \partial \vartheta_{i}=\operatorname{det} A_{i}$, where $A_{i}$ is the cofactor matrix of the element $\vartheta_{i}$. Lemma 2.1 is then proved since $\operatorname{det} \Gamma_{k}=1$ if $k$ is even and 0 otherwise.

We now consider objects in $\mathscr{L}$ called dimers which consist of a bond and its end points. To each non-overlapping collection of dimers in $\mathscr{L}$, the set $X$ of sites covered by this collection is such that (2.3) is nonvanishing. Conversely, to each $X \subseteq \mathscr{L}$ whose regular partition $\left(X_{1}, \ldots, X_{n}\right)$ is such that (2.3) is different from 0 , there exists a unique dimer configuration with $X$ being its covered set. This one-toone correspondence leads us to the following definition:

Definition 2.2. $X$ is a compatible set of sites if it coincides with the covered set of a dimer configuration in $\mathscr{L}$.

We have proven the following theorem:

Theorem 2.3.

$$
\operatorname{det}\left(\Gamma_{L}+(\zeta / \sqrt{N}) \Phi_{L}\right)=\sum_{\text {compatible } X} \prod_{j \in X^{c}}(\zeta / \sqrt{N}) \phi_{j}
$$

is the generating function of a stochastic monomer-dimer problem on $\mathscr{L}$ whose monomer activity at site $i$ is given by the Gaussian random variable $(\zeta / \sqrt{N}) \phi_{i}$.

The remainder of the paper is based on Eq. (2.4).

\section{Mean Field and Peierls Estimate}

Proof of Proposition 1.1. By setting $\phi_{i}=\varphi, i=1, \ldots, L$ in Eq. (2.4), the effective potential $V_{L}$, given by (1.11), can be written as

$$
V_{L}(\varphi)=N\left\{\frac{1}{2 N} \varphi^{2}-\frac{2}{L} \ln f_{L}((\zeta / \sqrt{N}) \varphi)\right\},
$$

where

$$
f_{L}(z)=\sum_{\text {compatible } X} z^{\left|X^{c}\right|} .
$$

The critical points of (3.1) are of the form $\varphi^{*}=m \sqrt{N} / \zeta$ with $m$ satisfying the self-consistency Eq. (1.10) which can be written as

$$
\frac{m^{2}}{2 \zeta^{2}}=\frac{1}{f_{L}(m)} \sum_{X} \frac{\left|X^{c}\right|}{L} m^{\left|X^{c}\right|} .
$$


Since $L$ is an even number, $f_{L}(z)$ is an even polynomial in $z$. This implies $V(-\varphi)=V(\varphi)$ and item (i) of Proposition 1.1.

In order to prove items (ii) and (iii), we shall find the set of all solutions of (3.3) and determine their stability.

Equation (3.3) can be written as

$$
\frac{m^{2}}{2 \zeta^{2}}=E_{L, m}(X)
$$

Here $E_{L, m}(\mathrm{X})$ is the mean of a discrete probability distribution on $[0,1]$ :

$$
\operatorname{Pr}_{L, m}(X=l / L)=\frac{1}{f_{L}(m)} C_{L}(l) m^{l}, \quad l=0,1, \ldots, L
$$

and $C_{L}(l)$ is a combinatorial factor which accounts for the number of different ways of placing $(L-l) / 2$ dimers on a lattice of size $L$. Notice that $C_{L}(l)=0$ if $L+l$ is odd.

$C_{L}(l)$ satisfies the recurrence relation

$$
C_{L}(l)=C_{L-1}(l-1)+C_{L-2}(l),
$$

since the left-most site is occupied either by a monomer or a dimer. Note also that

$$
C_{L}(L)=1 \text {. }
$$

It follows that

$$
C_{L}(l)=\left\{\begin{array}{cl}
\left(\begin{array}{c}
(L+l) / 2 \\
l
\end{array}\right) & \text { if } L+l \text { is even } \\
0 & \text { otherwise }
\end{array} .\right.
$$

From this one can see that

1. $E_{L, m}(\mathrm{X})$ increases monotonically from 0 to 1 as $m$ increases from 0 to infinity;

2. for small $m$,

$$
E_{L, m}(X)=m^{2}(L+2) / 4+O\left(m^{4}\right) .
$$

Therefore the self-consistency Eq. (1.10) has a unique solution if $\zeta^{2}>2$ / $(L+2)$. Since the correction to the leading term in $(3.7)$ is negative, one can show there is no solution if $\zeta^{2}<2 /(L+2)$. This proves (ii).

To prove (iii) we use Stirling's formula for any $l \in\{1, \ldots, L\}$ to get:

$$
\frac{1}{L} \ln C_{L}(l)=\frac{L+l}{2 L} \ln \frac{L+l}{2 L}-\frac{l}{L} \ln \frac{l}{L}-\frac{L-l}{2 L} \ln \frac{L-l}{2 L}+O\left(\frac{\ln L}{L}\right)
$$

as $L \rightarrow \infty$.

Therefore

where

$$
C_{L}(l) m^{l}=\exp \left\{L I_{m}(l / L)+O(\ln L)\right\}
$$

$$
I_{m}(x)=\frac{1+x}{2} \ln \frac{1+x}{2}-x \ln \frac{x}{m}-\frac{1-x}{2} \ln \frac{1-x}{2}
$$


is a concave function with maximum at $\hat{x}$ determined by the equation

$$
\frac{4 \hat{x}^{2}}{m^{2}\left(1-\hat{x}^{2}\right)}=1 \text {. }
$$

The Laplace asymptotic method can now be used to approximate the probability distribution of $X$ :

$$
\begin{aligned}
f_{L}(m)=\sum_{l=0}^{L} C_{L}(l) m^{l} & \sim \frac{L}{2} \int_{-\infty}^{\infty} \exp \left\{L I_{m}(x)\right\} d x \\
& \sim\left[\frac{\pi L}{2\left|I^{\prime \prime}(\hat{x})\right|}\right]^{1 / 2} \exp \left\{L I_{m}(\hat{x})\right\}
\end{aligned}
$$

and

$$
E_{L, m}(\mathrm{X})=\frac{1}{f_{L}(m)} \sum_{l=0}^{L} \frac{l}{L} C_{L}(l) m^{l} \sim \hat{x} .
$$

In these formulas, the fractional error is $O(1 / L)$.

The self-consistency Eq. (3.4) can thus be written as

$$
\frac{m^{2}}{2 \zeta^{2}}=\left(\frac{m^{2}}{4+m^{2}}\right)^{\frac{1}{2}}
$$

which has one unstable solution $m_{0}=0$ and two stable solutions

$$
m_{ \pm}= \pm\left[2\left(1+\zeta^{4}\right)^{1 / 2}-2\right]^{1 / 2}
$$

The profile of $V(\varphi)$, in the limit as $L \rightarrow \infty$, follows from the expression (see Eq. (3.1))

$$
\lim _{L \rightarrow \infty} \frac{1}{L} \ln f_{L}((\zeta / \sqrt{N}) \varphi)=\sup _{x \in[0,1]} I_{(\zeta / \sqrt{N}) \varphi}(x)=\frac{1}{2} \ln \frac{1+\hat{x}(\varphi)}{1-\hat{x}(\varphi)},
$$

where $\hat{x}(\varphi)=\left(1+\frac{4 N}{\zeta^{2} \varphi^{2}}\right)^{-1 / 2}$. This diverges logarithmically as $\varphi \rightarrow \pm \infty$ leading to a parabolic growth of $V(\varphi)$ for large $\varphi$. For small $\varphi$, the logarithm dominates the quadratic term and $V(\varphi) / N \simeq-2 \hat{x}(\varphi) \simeq-(\zeta / \sqrt{N})|\varphi|$ approaches 0 from negative values. $V(\varphi)$ attains to its minimum value at $\pm \varphi^{*}=m_{ \pm} \sqrt{N} / \zeta$ which, by (3.11), behaves as

$$
\varphi^{*} \simeq\left\{\begin{array}{ll}
\sqrt{N} \zeta & \text { for } \zeta \text { small } \\
\sqrt{2 N} & \text { for } \zeta \text { large }
\end{array} .\right.
$$

This implies

$$
V\left(\varphi^{*}\right) \simeq \begin{cases}-N \zeta^{2} & \text { for } \zeta \text { small } \\ -2 N\left(\ln 2 \zeta^{2}-1\right) & \text { for } \zeta \text { large }\end{cases}
$$

which concludes the proof of Proposition 1.1.

Proof of Proposition 1.2. Each Ising-like configuration, $\left\{\sigma_{i}\right\}_{i=1}^{L}$, determines a sequence $\left\{\ell_{i}\right\}_{i=1}^{p+1}$ of integers with $\sum_{i=1}^{p+1} \ell_{i}=L$, corresponding to the number of sites between two successive flips $\left(\ell_{1}\right.$ and $\ell_{p+1}$ being the number of sites from the boundary to the closest flip). 
From Eq. (2.4)

$$
f_{L}(\sigma)=\sum_{\text {compatible } X} \prod_{i \in X^{c}} \sigma_{i} .
$$

For each compatible set $X$ we distinguish dimers, whose covering set is $X$, according to whether they occupy flip positions or not. By summing non-flip dimers first, (3.14) can be written as

$$
f_{L}(\sigma)=\sum_{\mathbf{n}} f_{\ell_{1}-n_{1}}\left(m_{+}\right) f_{\ell_{2}-n_{2}-n_{1}}\left(m_{-}\right) \cdots f_{\ell_{p}-n_{p}-n_{p-1}}\left(m_{-}\right) f_{\ell_{p+1}-n_{p}}\left(m_{+}\right),
$$

where the summation is over all $p$-tuples $\mathbf{n}=\left(n_{1}, \ldots, n_{p}\right) \in\{0,1\}^{p}$ with $n_{j}=1$ if the position of the $j^{\text {th }}$ flip is occupied by a dimer and 0 otherwise. Notice that

$$
f_{k}\left(m_{-}\right)=(-1)^{k} f_{k}\left(m_{+}\right) \text {. }
$$

The full proof at this stage continues with an elementary cluster expansion argument to deal with the ratio of two partition functions. Since the result is not of direct interest to the main body of the paper, this somewhat long argument will be omitted.

To give instead a simple argument which needs only the binomial theorem, let us assume

$$
\frac{f_{\ell_{0}-n}(m)}{f_{\ell_{0}}(m)}=\left(\frac{2 \hat{x}}{m(1+\hat{x})}\right)^{n}
$$

with $\hat{x}$ given by (3.8). This is asymptotically true when either $\ell_{0} \rightarrow \infty$ or $m \rightarrow \infty$. Equation (3.17) can be obtained either from (3.9) or by iteratively solving the equation

$$
f_{\ell}(m)=m f_{\ell-1}(m)+f_{\ell-2}(m)
$$

for $\Delta_{\ell}=f_{\ell}(m) / f_{\ell-1}(m)$.

In view of (3.16), (3.17) and (3.8), (3.15) can be written as

$$
\begin{aligned}
\frac{f_{L}(\sigma)}{\prod_{j=1}^{p+1} f_{\ell}(m)} & =(-1)^{\ell_{2}+\ell_{4}+\cdots+\ell_{p}}\left\{\sum_{n=0,1}\left(\frac{-4 \hat{x}^{2}}{m^{2}(1+\hat{x})^{2}}\right)^{n}\right\}^{p} \\
& =(-1)^{\ell_{2}+\ell_{4}+\cdots+\ell_{p}}\left(1-\frac{1-\hat{x}}{1+\hat{x}}\right)^{p} .
\end{aligned}
$$

Similarly,

$$
\frac{f_{L}(m)}{\prod_{j=1}^{p+1} f_{\ell_{j}}(m)}=\left(1+\frac{1-\hat{x}}{1+\hat{x}}\right)^{p} .
$$

Equations (3.18) and (3.19) lead to

$$
\left|\frac{f_{L}(\sigma)}{f_{L}(m)}\right|=\hat{x}^{p}
$$

with $\hat{x}$, from (3.8) and (3.11), strictly less than 1 for any $0<\zeta<\infty$.

The cluster expansion argument converges for large $m$, and agrees with (3.20):

$$
\left|\frac{f_{L}(\sigma)}{f_{L}(m)}\right| \leqq\left[\hat{x}\left(1+O\left(1 / m^{6}\right)\right)\right]^{p} .
$$


Proof of Proposition 1.3. We start by writing the two-point function (1.13) in the Matthews-Salam representation. Using (1.5)-(1.7) with (1.6) replaced by

$$
\begin{gathered}
\int \prod_{z \in \Lambda} d \bar{\psi}_{z} d \psi_{z} \bar{\psi}_{x} \cdot \psi_{y} \exp \left\{\sum_{z \in \Lambda} \bar{\psi}_{z} \cdot[(i a \mathbf{D}+(\zeta / \sqrt{N}) \boldsymbol{\Phi}) \psi]_{z}\right\} \\
=2 N\left(\Gamma_{L}+(\zeta / \sqrt{N}) \Phi_{L}\right)_{k l}^{-1} \operatorname{det}\left(\Gamma_{L}+(\zeta / \sqrt{N}) \Phi_{L}\right)^{2 N} ; \\
k=x / a, \quad l=y / a,
\end{gathered}
$$

and recalling (1.1) and (1.3), the two-point function can be written as

$$
\left\langle\bar{\psi}_{x} \cdot \psi_{y}\right\rangle_{L}=2 N \int \prod_{i=1}^{L} d \phi_{i} g_{L}(\phi)\left(\Gamma_{L}+(\zeta / \sqrt{N}) \Phi_{L}\right)_{k l}^{-1}
$$

(We have used the evenness of $L$ in (3.21) to write $\left(-\Gamma_{L}+\vartheta_{L}\right)_{k l}^{-1}=\left(\Gamma_{L}+\vartheta_{L}\right)_{k l}^{-1}$.)

In the mean field approximation $\phi_{i}=\varphi, i=1, \ldots, L$ and the density $g_{L}$ is replaced by $h_{L}$. The mean field two-point function is then given by

$$
\left\langle\bar{\psi}_{x} \cdot \psi_{y}\right\rangle_{L}=2 N \int d \varphi h_{L}(\varphi)\left(\Gamma_{L}+(\zeta / \sqrt{N}) \varphi 1_{L}\right)_{k l}^{-1}
$$

which can be written, from (1.12), as

$$
\left\langle\bar{\psi}_{x} \cdot \psi_{y}\right\rangle_{L}=2 N\left(\Gamma_{L}+m 1_{L}\right)_{k l}^{-1}+O(1 / L),
$$

where $m=m_{+}$is given by (3.11).

To avoid boundary influences, it is convenient to replace $\mathscr{L}$ by $\mathscr{L}^{\prime}=$ $[-L / 2+1, L / 2] \cap \mathbf{Z}$. The inverse matrix in (3.23) can be readily evaluated by the method of cofactors. It is given by

$$
\left(\Gamma_{L}+m 1_{L}\right)_{k l}^{-1}=(-1)^{k+l} \frac{f_{L / 2+k-1}(m) f_{L / 2-l-1}(m)}{f_{L}(m)} .
$$

We now use the Laplace asymptotic method to evaluate the right-hand side of (3.24). In the limit as $L \rightarrow \infty$, expression (3.9) can be used to obtain the following behavior:

$$
\begin{aligned}
\left(\Gamma_{L}+m 1_{L}\right)_{k l}^{-1} & =(-1)^{k+l} e^{-|l-k| I_{m}(\hat{x})}+O(1 / L) \\
& =(-1)^{k+l}\left(\frac{1-\hat{x}}{1+\hat{x}}\right)^{|l-k| / 2}+O(1 / L),
\end{aligned}
$$

which, by using (3.8) and (3.11), gives

$$
\left(\Gamma_{L}+m 1_{L}\right)_{k l}^{-1}=(-1)^{k+l}\left[\left(1+\zeta^{4}\right)^{1 / 2}-\zeta^{2}\right]^{|l-k| / 2}+O(1 / L) .
$$

The proof of Proposition 1.3 follows from (3.23) and (3.25).

\section{Proof of Theorem 1.4}

Asymptotic analysis and a transfer matrix technique will now be combined to evaluate the partition function $Z_{L}$. 
Let $\mu_{L, 0}$ be the free measure given by (1.1) with $\zeta=0$ and notice that $\mu_{L, 0}(\phi)=\prod_{j} \mu_{0}\left(\phi_{j}\right)$ is a product measure with

$$
d \mu_{0}(\phi)=\frac{1}{\sqrt{2 \pi}} e^{-\frac{1}{2} \phi^{2}} d \phi
$$

(recall det $\Gamma_{L}=1$ ).

Inserting the determinant expansion (2.4) into (1.3), we have

$$
Z_{L}=\sum_{\mathbf{X}} \int d \mu_{L, 0}(\phi) \prod_{i=1}^{2 N}\left\{\prod_{y \in X_{i}^{c}}(\zeta / \sqrt{N}) \phi_{y}\right\}
$$

where $\mathbf{X}=\left(X_{1}, \ldots, X_{2 N}\right)$ runs over all collections of $2 N$ compatible sets.

Performing the Gaussian integrals in (4.1), this equation can be written as

$$
\begin{aligned}
Z_{L}= & \sum_{\mathbf{n} \in \mathcal{N}_{L}} \delta_{n_{L}, 0}\left(\begin{array}{c}
2\left(N-n_{L}\right) \\
2 n_{1}
\end{array}\right)\left(\begin{array}{c}
2\left(N-n_{1}\right) \\
2 n_{2}
\end{array}\right) \cdots\left(\begin{array}{c}
2\left(N-n_{L-1}\right) \\
2 n_{L}
\end{array}\right) \\
& \times \alpha\left(2\left(N-n_{1}-n_{L}\right)\right) \alpha\left(2\left(N-n_{2}-n_{1}\right)\right) \cdots \alpha\left(2\left(N-n_{L}-n_{L-1}\right)\right),
\end{aligned}
$$

where

$$
\mathscr{N}_{L}=\left\{\mathbf{n} \in\{0,1, \ldots, N\}^{L}: 0 \leqq n_{k+1}+n_{k} \leqq N, k=1, \ldots, L-1\right\}
$$

and

$$
\alpha(k)=\int d \mu_{0}(\phi)\left(\frac{\zeta}{\sqrt{N}} \phi\right)^{k}=\left\{\begin{array}{cl}
0 & \text { if } k \text { is odd } \\
\left(\frac{\zeta^{2}}{2 N}\right)^{k / 2} \frac{k !}{(k / 2) !} & \text { if } k \text { is even }
\end{array} .\right.
$$

In expression (4.2), the occupation numbers $\mathbf{n}=\left(n_{1}, \ldots, n_{L}\right)$ count how many pairs of copies of each dimer there are in the collection $\left\{X_{i}\right\}$. To each configuration n a weight is associated due to the integration (4.3) at each site (the second line in (4.2)). The sum over $\mathbf{X}$ with $\mathbf{n}$ held fixed is counted by the binomial factors. The Kronecker delta $\delta_{n_{L}, 0}$ enforces the open boundary condition at each end.

Expression (4.2) can be symmetrized and written in the following compact form:

$$
Z_{L}=\alpha^{L} \operatorname{Tr}\left(P_{0} T^{L}\right) .
$$

Here $\alpha=\alpha(2 N), P_{0}$ is the projection $\left|\delta_{0}\right\rangle\left\langle\delta_{0}\right|$, and $T$ is a $(N+1) \times(N+1)$ symmetric matrix whose elements are given by

$$
T\left(n, n^{\prime}\right)=S(n) R\left(n, n^{\prime}\right) S\left(n^{\prime}\right)
$$

with

and

$$
S(n)=\left(\frac{2 N^{2}}{e \zeta^{2}}\right)^{n}\left(\frac{(2(N-n)) !}{(2 N) !(2 n) !}\right)^{1 / 2}
$$

$$
R\left(n, n^{\prime}\right)=\left(\frac{e}{N}\right)^{n+n^{\prime}} \frac{N !}{\left(N-n-n^{\prime}\right) !} \chi\left(n+n^{\prime} \leqq N\right),
$$

where $\chi(P)=1$ if the relation $P$ is satisfied and 0 otherwise. 
Theorem 1.4 will follow from the usual transfer matrix method: let $\left\{\lambda_{j}, \psi_{j}\right\}_{j=0}^{N}$ be the eigenvalues and eigenvectors of $T$ listed in order of decreasing absolute value: $\left|\lambda_{j}\right| \geqq\left|\lambda_{j+1}\right|$. Let us assume that $\lambda_{0}>\bar{\lambda}=\sup _{j \geqq 1}\left|\lambda_{j}\right|$. The partition function can thus be written as

$$
Z_{L}=\alpha^{L} \sum_{i=0}^{N}\left\langle\delta_{0} \mid \psi_{i}\right\rangle^{2} \lambda_{i}^{L}=\left\langle\delta_{0} \mid \psi_{0}\right\rangle^{2}\left(\alpha \lambda_{0}\right)^{L}\left[1+O\left(\left(\bar{\lambda} / \lambda_{0}\right)^{L}\right)\right]
$$

The eigenvalues of $T$ are given by the following result which will be proven using asymptotic analysis.

Lemma 4.1. For any $0<\zeta<\infty$, there exist $N_{0}=N_{0}(\zeta), n_{0}=n_{0}(\zeta)$ and $c_{0}=c_{0}(\zeta)$ such that, for $N>N_{0}$, the first $n_{0}$ eigenvalues of $T$ can be written in the following form:

$$
\lambda_{j}=c_{0} e^{N \bar{f}}\left(\mu_{j}^{(0)}+O\left(\frac{1}{N^{1 / 2}}\right)\right), \quad j=0,1, \ldots, n_{0},
$$

$n_{0}$ is chosen so that $\left|\mu_{i}^{(0)}-\mu_{j}^{(0)}\right| \leqq O(1)$ for all $i \neq j \leqq n_{0}$. Here $\bar{f} \equiv \max _{(x, y) \in \mathscr{I}}$ $f(x, y)=f(\bar{x}, \bar{x})$ is defined in Theorem 1.4. The quantities $\mu_{0}^{(0)}=1$ and $\mu_{j}^{(0)}$, $j=1, \ldots, N$, are determined by an associated integral equation (4.16). Moreover

$$
\lambda_{0}>-\lambda_{1}>\lambda_{2}>\cdots>(-1)^{n_{0}} \lambda_{n_{0}}>\sup _{n>n_{0}}\left|\lambda_{n}\right| .
$$

Remark. We only really need (4.7) for $j=0,1$.

Conclusion of Proof of Theorem 1.4. In view of (4.3) and Lemma 4.1, (4.6) can be written as

$$
Z_{L}=\left\langle\delta_{0} \mid \psi_{0}\right\rangle^{2}\left[c_{0} e^{\nu N}(1+O(1 / N))\right]^{L}\left[1+O\left(\left(\mu_{1}^{(0)}\right)^{L}\right)\right]
$$

with

$$
v=\bar{f}-\ln \frac{e}{2 \zeta^{2}}=\ln \frac{(1-\bar{x})}{\bar{x}}>0
$$

for any $0<\zeta<\infty$ (recall $0<\bar{x}<1 / 2$ by (1.18)). This concludes the proof of Theorem 1.4.

Lemma 4.1 will be proved by finding a workable continuum approximation to the matrix $T$. We begin by writing an asymptotic expansion in $1 / N$. In order to apply Stirling's formula to expression (4.5) it is convenient to introduce a set $\mathscr{I}_{N, M} \in(1 / N \mathbf{Z})^{2}$ given by

$$
\mathscr{I}_{N, M}=\left\{\left(z, z^{\prime}\right) \in[1 / M, 1]^{2} \cap(1 / N \mathbf{Z})^{2}: z+z^{\prime} \leqq 1-1 / M\right\},
$$

where $M$ will be taken depending on $N$ with $M / N \rightarrow c$ as $N \rightarrow \infty$ for some $0<c<1 / 2$. We set $\mathscr{I}_{N}=\mathscr{I}_{N, \infty}$ and notice that $\mathscr{I}_{N, M}$ excludes points closer than $1 / M$ to the border of $\mathscr{I}_{N}$.

Writing $\left(n, n^{\prime}\right)=(N x, N y), M$ can be chosen such that if $(x, y) \in \mathscr{I}_{N, M}$,

$$
K !=\sqrt{2 \pi K} e^{-K} K^{K}\left(1+\frac{1}{12 K}+O\left(\frac{1}{N^{2}}\right)\right)
$$


with $K$ being any of the functions $N x, N y$ or $N(1-x-y)$. The transfer matrix (4.5) can thus be written as

$$
T(N x, N y)=\frac{1}{\sqrt{4 \pi N}} L(x, y) \exp \{N f(x, y)\}\left(1+\frac{l(x, y)}{N}+O\left(\frac{1}{N^{2}}\right)\right),
$$

where $f$ is given by (1.7),

and

$$
L(x, y)=\left(\frac{1-x}{x}\right)^{\frac{1}{4}}\left(\frac{1}{1-x-y}\right)^{\frac{1}{2}}\left(\frac{1-y}{y}\right)^{\frac{1}{4}}
$$

$$
l(x, y)=\frac{1}{12}\left[\frac{1}{4 x(1-x)}+\frac{1}{1-x-y}+\frac{1}{4 y(1-y)}\right] .
$$

The next proposition gives us properties of $f(x, y)$ which will show that $T$ can be approximated for large $N$ by an integral transfer operator.

Proposition 4.2. Let $f$ be given by (1.17). It follows that

(i) the critical points of $f$ lie on the circle with centre $\left(\left(1+\zeta^{-2}\right) / 2,(1+\right.$ $\left.\zeta^{-2}\right) / 2$ ) and radius $\left(\left(1+\zeta^{-4}\right) / 2\right)^{1 / 2}$

(ii) $f$ is a symmetric concave function on the domain $\mathscr{I} \equiv \mathscr{I}_{\infty}$. It attains its maximum at $(\bar{x}, \bar{x}) \in \mathscr{I}$, where $\bar{x}$ is the solution (1.18) of

$$
2 \zeta^{2} \frac{\bar{x}(1-\bar{x})}{1-2 \bar{x}}=1
$$

which is given asymptotically by

$$
\bar{x} \sim\left\{\begin{array}{ll}
\left(1 / 2 \zeta^{2}\right)\left[1-\left(1 / 2 \zeta^{2}\right)\right] & \zeta \gg 1 \\
1 / 2\left(1-\zeta^{2} / 2\right) & \zeta \ll 1
\end{array} .\right.
$$

(Notice that $(\bar{x}, \bar{x}) \in \mathscr{I}_{N, M}$, provided $2 / M<\zeta^{2}<M / 2$ );

(iii) the Hessian of $f$ at $(x, y)$ is given by

$$
\mathbf{h}(x, y)=\frac{-1}{1-x-y}\left(\begin{array}{cc}
1+\frac{(1-2 x)(1-x-y)}{x(1-x)} & 1 \\
1 & 1+\frac{(1-2 y)(1-x-y)}{y(1-y)}
\end{array}\right) .
$$

Remark. Plugging this result into Eq. (4.9), and retaining leading order in $1 / N$ implies that

$$
T\left(n, n^{\prime}\right)=N^{-1 / 2} c_{0} e^{N \bar{f}} \mathscr{A}\left(z_{n}, z_{n^{\prime}}\right)(1+O(1 / N)),
$$

where

$$
z_{n}=n / \sqrt{N}-\sqrt{N} \bar{x}
$$

and the kernel $\mathscr{A}$ is given by

$$
\mathscr{A} v(z)=\sqrt{\frac{\rho}{2 \pi}} \int_{-\infty}^{\infty} e^{\frac{1}{2} q\left(z, z^{\prime}\right)} v\left(z^{\prime}\right) d z^{\prime}
$$

$q$ is the (negative definite) quadratic form defined by the Hessian matrix (4.13) at the critical point, $\overline{\mathbf{h}} \equiv \mathbf{h}(\bar{x}, \bar{x})$, whose entries $\overline{\mathbf{h}}_{i j}, i, j=1,2$, are given by

$$
-\overline{\mathbf{h}}_{11}=-\overline{\mathbf{h}}_{22}=-\overline{\mathbf{h}}_{12}+2 \zeta^{2}=-\overline{\mathbf{h}}_{21}+2 \zeta^{2}=\frac{1}{1-2 \bar{x}}+2 \zeta^{2} .
$$


The values

and

$$
c_{0}=\frac{1}{\sqrt{2 \rho}} L(\bar{x}, \bar{x})=\frac{1}{2 \rho^{1 / 2} \zeta \bar{x}}
$$

$$
\begin{aligned}
\rho & =-\overline{\mathbf{h}}_{11}+\sqrt{\operatorname{det} \overline{\mathbf{h}}} \\
& =\frac{\zeta^{2}}{\left(1+\zeta^{4}\right)^{1 / 2}-1}\left\{2\left(1+\zeta^{4}\right)^{1 / 2}\left[1+\left(1-\left(1+\zeta^{4}\right)^{-1 / 2}\right)^{1 / 2}\right]-1\right\}
\end{aligned}
$$

are defined for convenience.

Proof of Proposition 4.2. Items (i) and (iii) follow by simple calculation. It follows from (4.13) that $f$ is concave if and only if

$$
(1-2 x) y(1-y)+(1-2 y) x(1-x)+(1-2 x)(1-2 y)(1-x-y)>0
$$

holds. This condition is fulfilled for all $(x, y) \in \mathscr{I}$ since, by reorganizing (4.18), it can be written as

$$
(1-x-y+2 x y)(1-x-y) .
$$

This shows that $\mathbf{h}$ is a negative definite matrix, proving item (ii) and Proposition 4.2.

The next proposition gives a complete characterization of the spectrum $\left\{\mu_{j}^{(0)}\right\}_{j=0}^{\infty}$ and eigenfunctions of the continuum operator $\mathscr{A}: L^{2}(\mathbf{R}) \rightarrow L^{2}(\mathbf{R})$.

Proposition 4.3 (see e.g. [CE]). $\mathscr{A}$ is a self-adjoint compact operator in $L^{2}(\mathbf{R})$. The spectrum $\sigma(\mathscr{A})$ of $\mathscr{A}$ consists of the sequence

$$
\mu_{j}^{(0)} \equiv\left(-\left|\overline{\mathbf{h}}_{12}\right| / \rho\right)^{j}, \quad j=0, \ldots, \infty
$$

and the corresponding eigenfunctions,

$$
\psi_{j}(z)=\left(\frac{\gamma}{2^{j} j ! \sqrt{\pi}}\right)^{1 / 2} \exp \left\{-\frac{1}{2} \gamma^{2} z^{2}\right\} H_{j}(\gamma z), \quad j=0,1, \ldots,
$$

where

$$
\gamma^{2}=\frac{\rho}{2}\left(1-\left(\overline{\mathbf{h}}_{12} / \rho\right)^{2}\right)=\sqrt{\operatorname{det} \overline{\mathbf{h}}},
$$

and $H_{j}$ is the $j^{\text {th }}$ normalized Hermite polynomial, are orthonormal with respect to the inner product $\langle\cdot, \cdot\rangle$ in $L^{2}(\mathbf{R}, d x)$ with weight 1 , i.e.,

$$
\left\langle\psi_{n}, \mathscr{A} \psi_{m}\right\rangle=\mu_{m}^{(0)} \delta_{n m} .
$$

Proof of Lemma 4.1. We shall use a Rayleigh-Ritz argument to show that the largest eigenvalues of $T$ are approximated to order $N^{-1 / 2}$ by the largest eigenvalues of $\mathscr{A}$. The discrete operator $A=c_{0}^{-1} e^{-N \bar{f}} T: \mathbf{R}^{N+1} \rightarrow \mathbf{R}^{N+1}$ is approximated by the continuum operator $\mathscr{A}: L^{2}(\mathbf{R}) \rightarrow L^{2}(\mathbf{R})$. To establish this, we introduce an isometry of the vector space $\mathbf{R}^{N+1}$ into piecewise constant functions in the vector space $L^{2}(\mathbf{R})$. 
Define intervals

$$
J_{n}=\left(\frac{n-1 / 2}{\sqrt{N}}-\sqrt{N} \bar{x}, \frac{n+1 / 2}{\sqrt{N}}-\sqrt{N} \bar{x}\right]
$$

with centres $z_{n}=n / \sqrt{N}-\sqrt{N} \bar{x}$, for $n=0,1, \ldots, N$, such that $J=\bigcup_{n=0}^{N} J_{n}$ is the interval $\left(-\frac{1}{2 N}, 1+\frac{1}{2 N}\right]$, translated by $\bar{x}$, then dilated by $\sqrt{N}$. Then, for $u \in \mathbf{R}^{N+1}$, let

The adjoint $i^{\dagger}$ is given by

$$
(i(u))(z)=\left\{\begin{array}{ll}
N^{1 / 4} u(n) & z \in J_{n} \\
0 & z \notin \bigcup_{n} J_{n}
\end{array} .\right.
$$

Note that $i^{\dagger} i$ is the identity matrix.

$$
\left(i^{\dagger}(v)\right)(n)=N^{1 / 4} \int_{J_{n}} v(z) d z
$$

The operator kernel of $i A i^{\dagger}$ is a step function with step-width $1 / \sqrt{N}$, which we will see in the next lemma approximates $\mathscr{A}\left(z, z^{\prime}\right)$ in the operator norm.

Lemma 4.4. 1. The operator norm of the error $\Delta A=i A i^{\dagger}-\mathscr{A}$ is bounded by

$$
\|\Delta A\|=O(1 / \sqrt{N}) .
$$

2. There is some value $n_{0}=n_{0}(\zeta) \gg 1$ such that for each eigenfunction $\psi_{j}$, $j=0,1, \ldots, n_{0}$ of $\mathscr{A}$, we have

$$
\left\|\left(1-i i^{\dagger}\right) \psi_{j}\right\| \leqq O\left(N^{-1 / 4}\right) .
$$

Proof. Introduce the functions

$$
[z]=z_{n}, \quad \text { if } z \in J_{n}
$$

and

$$
\xi(z)=z-[z], \quad \vec{\xi}\left(z, z^{\prime}\right)=\left(z, z^{\prime}\right)-\left([z],\left[z^{\prime}\right]\right)
$$

defined for $z, z^{\prime} \in J$. We note that (4.14) implies that there is a constant $\alpha=O(1)>0$ such that for $\vec{z}=\left(z, z^{\prime}\right) \in N^{1 / 2} \mathscr{I}_{N, M}$,

$$
\left|\mathscr{A}\left(z, z^{\prime}\right)-\mathscr{A}\left([z],\left[z^{\prime}\right]\right)\right| \leqq O(1)|\vec{\xi}| e^{-\alpha|\vec{z}|^{2}}
$$

and

$$
\left|\mathscr{A}\left([z],\left[z^{\prime}\right]\right)-\left(i A i^{\dagger}\right)\left(z, z^{\prime}\right)\right| \leqq O(1 / N) e^{-\alpha|\vec{z}|^{2}},
$$

while for $\left(z, z^{\prime}\right) \notin N^{1 / 2} \mathscr{I}_{N, M}$, one has

$$
\left|\mathscr{A}\left(z, z^{\prime}\right)-\left(i A i^{\dagger}\right)\left(z, z^{\prime}\right)\right| \leqq e^{-\alpha|\vec{z}|^{2}} .
$$

These facts serve to establish an estimate of the $L^{2}$-norm of the kernel $\triangle A\left(z, z^{\prime}\right)$ sufficient to imply item (1) of the lemma.

For (2), take $n_{0}(\zeta)$ small enough that the estimate

$$
\left|\nabla \psi_{j}(z)\right| \leqq O(1) e^{-\alpha|z|^{2}}
$$


holds for all $j \leqq n_{0}$. Then,

$$
\begin{aligned}
\left\langle\left(1-i i^{\dagger}\right) \psi_{j},\left(1-i i^{\dagger}\right) \psi_{j}\right\rangle & =\sum_{n} \int_{J_{n}} d z \bar{\psi}_{j}(z)\left[\psi_{j}(z)-\sqrt{N} \int_{J_{n}} \psi_{j}\left(z^{\prime}\right) d z^{\prime}\right] \\
& \leqq \sum_{n} \int_{J_{n}} d z\left|\bar{\psi}_{j}(z)\right|\left[O\left(N^{-1 / 2}\right) e^{-\alpha|z|^{2}}\right] \\
& \leqq O(1 / \sqrt{N}) .
\end{aligned}
$$

Using this, and the Rayleigh-Ritz formula, we next show upper and lower bounds on the eigenvalues $\mu_{j}$ of $A$ for $j \leqq n_{0}(\zeta)$. We choose trial eigenfunctions

$$
\varphi_{j}=c_{j} i^{\dagger} \psi_{j}
$$

with normalization $c_{j}^{-2}=\left(i^{\dagger} \psi_{j}, i^{\dagger} \psi_{j}\right)=\left\langle\psi_{j}, i i^{\dagger} \psi_{j}\right\rangle$. From Lemma 4.4,

$$
c_{j}^{-2}=1+\left\langle\left(1-i i^{\dagger}\right) \psi_{j},\left(1-i i^{\dagger}\right) \psi_{j}\right\rangle=1+O(1 / \sqrt{N}) .
$$

For $j \leqq n_{0}$ even, further use of Lemma 4.4 gives

$$
\begin{aligned}
\mu_{j} & =\inf _{\left\{\hat{\varphi}_{0}, \hat{\varphi}_{2}, \ldots, \hat{\varphi}_{J-2}\right\}} \sup _{\varphi \in\left[\hat{\varphi}_{0}, \hat{\varphi}_{2}, \ldots, \hat{\varphi}_{j-2}\right]^{\perp}} \frac{(\varphi, A \varphi)}{(\varphi, \varphi)} \\
& \leqq \sup _{\varphi \in\left[\varphi_{0}, \varphi_{2}, \ldots, \varphi_{J-2}\right]^{\perp}} \frac{(\varphi, A \varphi)}{(\varphi, \varphi)} \\
& =\sup _{\psi \in i\left[\varphi_{0}, \varphi_{2}, \ldots, \varphi_{J-2}\right]^{\perp}} \frac{\left\langle\psi, i A i^{\dagger} \psi\right\rangle}{\langle\psi, \psi\rangle} \\
& \leqq \sup _{\psi \in\left[\psi_{0}, \psi_{2}, \ldots, \psi_{J-2}\right]^{\perp}} \frac{\left\langle\psi, i A i^{\dagger} \psi\right\rangle}{\langle\psi, \psi\rangle} \\
& =\mu_{j}^{(0)}+O(1 / \sqrt{N}) .
\end{aligned}
$$

bound (4.33) is obtained by taking a specific set of vectors $\left\{\hat{\varphi}_{0}, \ldots, \hat{\varphi}_{j-2}\right\}$. Note that (4.35) is true since the sup in (4.34) is over a subset of the sup in (4.35). We obtain similar lower bounds for $j$ odd, by replacing $A$ by $-A$ above.

We also have a lower bound on $\mu_{0}$ :

$$
\mu_{0} \geqq\left(\varphi_{0}, A \varphi_{0}\right)=1+O(1 / \sqrt{N}) .
$$

Finally, we can prove lower bounds on $\mu_{j}, j>0$ even, and upper bounds for $j$ odd. We illustrate with $\mu_{2}$. From (4.36) and (4.37), it follows that a true $\mu_{0}$-eigenvector of $A$ can be written $\tilde{\varphi}_{0}=\varphi_{0}+\delta \varphi_{0}$, where $(\varphi, \delta \varphi)=0$ and $\|\delta \varphi\|^{2}=O(1 / \sqrt{N})$. Then

$$
\begin{aligned}
\mu_{2} & =\sup _{\varphi \in\left[\tilde{\varphi}_{0}\right]^{\perp}}(\varphi, A \varphi) \\
& \geqq \sup _{\varphi \in \operatorname{Span}\left(\varphi_{0}, \varphi_{2}\right) \cap\left[\tilde{\varphi}_{0}\right]^{\perp}}(\varphi, A \varphi) \\
& =\mu_{2}^{(0)}+O(1 / \sqrt{N}) .
\end{aligned}
$$


The last step follows because the maximizing $\varphi$ is of the form $\alpha \varphi_{0}+\beta \varphi_{2}$ with $\alpha^{2}=O(1 / \sqrt{N})$ and $\beta^{2}=1-O(1 / \sqrt{N})$.

Continuing the same argument gives lower (upper) bounds for $1<j \leqq n_{0}$ even (odd). This concludes the proof of Lemma 4.1.

\section{Higher Order $1 / N$ Corrections}

In this section we outline how to obtain a complete $1 / N$ expansion for the first eigenvalues of $T$. The main technical ingredient in our method is Temple's Inequality (for a proof see Problem 15, p. 365 of ref. [RS]):

Proposition 5.1. Let $A$ be a self-adjoint operator. Let $I=(a, b) \subset \mathbf{R}$ be an open interval and $\varphi$ be a normalized trial vector such that

and

$$
\sigma(A) \cap I=\{\mu\}
$$

$$
\eta<(\gamma-a)(b-\gamma)
$$

where $\gamma=(\varphi, A \varphi)$ and $\eta=\left(\varphi,(A-\gamma)^{2} \varphi\right)$. Then the following upper and lower bounds hold:

$$
\gamma-\frac{\eta}{(b-\gamma)}<\mu<\gamma+\frac{\eta}{(\gamma-a)} \text {. }
$$

Since by Lemma 4.1, we have succeeded in separating the first $n_{0}$ eigenvalues of $A$ into nonoverlapping intervals of width $O(1)$ (at least if $N_{0}$ is large), Temple's inequality applied to $A$ will allow us to improve the eigenvalue estimate (4.7) to any order in $1 / N$ provided we can systematically improve the approximate eigenfunctions. This can in fact be done, using Stirling's formula and the Laplace asymptotic method. One also needs results of the following type to estimate the error in replacing Riemann sums by Riemann integrals:

Proposition 5.2. Consider the interval $[a, b)=\bigcup_{j=1}^{N} I_{j}$, where $I_{j}=\left[x_{j}-\frac{\Delta x}{2}, x_{j}+\frac{\Delta x}{2}\right)$, $\Delta x=\frac{(b-a)}{N}$ and $x_{j}=a+(j-1 / 2) \Delta x$. Then for any $C^{4}$ function $f:$

$$
\begin{aligned}
& \left|\int_{a}^{b} f(x) d x-\frac{(\Delta x)^{2}}{3}\left[f^{\prime}(b)-f^{\prime}(a)\right]-\Delta x \sum_{j=1}^{N} f\left(x_{j}\right)\right| \\
& \quad \leqq O(1) \frac{(b-a)^{5}}{N^{4}}\left\|f^{(i v)}\right\|_{\infty} .
\end{aligned}
$$

Remarks.

1. This formula is obtained by Taylor expanding the integrand over each subinterval. The important point here is that by including boundary terms, the error estimate is improved compared to that of the midpoint rule. We shall apply this formula on the $O(\sqrt{N})$-width interval $J(4.21)$ to functions such as $\mathscr{A}\left(z, z^{\prime}\right)$ and $\psi_{j}(z)$ with $O(1)$ Gaussian decay. Thus the included boundary terms are $O\left(N^{-k}\right)$ for any $k$.

2. Similar arguments including further boundary terms, lead to more general formulas with error estimates of the form $O\left(N^{-k}\right)$ for arbitrarily large $k$. 
We illustrate how these ingredients combine to produce the $O(1 / \sqrt{N})$ correction to Lemma 4.1.

To derive an approximation for the matrix $A=c_{0}^{-1} e^{-N \bar{f}} T$ up to and including order $1 / \sqrt{N}$, we Taylor expand the functions $f, L$ and $l$ in (4.9) around the maximum value $(\bar{x}, \bar{x})$, rescaling the arguments by the factor $1 / \sqrt{N}$. This yields an integral kernel $\mathscr{A}^{1}\left(z, z^{\prime}\right)$ of the integral operator given by

$$
\mathscr{A}^{1}=\mathscr{A}+(1 / \sqrt{N}) \mathscr{V}_{1}
$$

where $\mathscr{A}$ is given (4.16) and $\mathscr{V}_{1}$ is the integral operator defined by

$$
\mathscr{V}_{1} u(z)=\frac{1}{2 \sqrt{\pi} c_{0}} \int_{-\infty}^{\infty} K_{1}\left(z, z^{\prime}\right) e^{\frac{1}{2} q\left(z, z^{\prime}\right)} u\left(z^{\prime}\right) d z^{\prime}
$$

with

$$
K_{1}\left(z_{1}, z_{2}\right)=\overline{d L} \cdot \mathbf{z}+\frac{\bar{L}}{3 !} d^{\overline{3}} f \cdot \mathbf{z}^{3} .
$$

Here, for $\mathbf{z}=\left(z_{1}, z_{2}\right)$ and $g: \mathbf{R}^{2} \mapsto \mathbf{R}$, we have

$$
d^{\bar{k}} g \cdot \mathbf{z}^{k}=\sum_{i_{1}, \ldots, i_{k} \in\{1,2\}^{k}} \frac{\partial^{k} g}{\partial z_{i_{1}} \cdots \partial z_{i_{k}}}(\bar{x}, \bar{x}) z_{i_{1}} \cdots z_{i_{k}} .
$$

To write the trial eigenfunctions $\varphi_{j}$ in Proposition 5.1 that allow us to use (5.2),we have to replace the operator $i^{\dagger}$ defined previously by a surjection $\Sigma$. For any function $v: \mathbf{R} \mapsto \mathbf{R}$, we define

$$
(\Sigma v)(n)=\frac{1}{N^{1 / 4}} v\left(z_{n}\right), \quad n=0, \ldots, N .
$$

Note that for piecewise constant functions in each $J_{n}, \psi(z)$,

$$
(\Sigma \psi, \Sigma \psi)=\langle\psi, \psi\rangle \text {. }
$$

We choose the trial functions given by

with $c_{j}^{-2}=\left(\Sigma \psi_{j}, \Sigma \psi_{j}\right)$.

$$
\varphi_{j}=c_{j} \Sigma \psi_{j}
$$

Now we use Temple's inequality. We choose trial intervals

$$
I_{j}=\left(\mu_{j}^{(0)}-O(1), \mu_{j}^{(0)}+O(1)\right)
$$

for $j \leqq n_{0}$ in such a way that Lemma 4.1 ensures there is exactly one eigenvalue $\mu_{j}$ in each $I_{j}$ (for this, $N_{0}$ should possibly be increased). We must find $\mu_{j}^{(1)}$ so that

$$
\gamma_{j}=\left(\varphi_{j}, A \varphi_{j}\right)=\mu_{j}^{(0)}+\frac{1}{\sqrt{N}} \mu_{j}^{(1)}+O(1 / N)
$$

and the variance $\eta_{j}=O(1 / N)$.

Now, using Stirling (4.9), (5.2) and the Laplace method we get

$$
\frac{\left(\Sigma \psi_{j}, A \Sigma \psi_{j}\right)}{\left(\Sigma \psi_{j}, \Sigma \psi_{j}\right)}=\frac{\left\langle\psi_{j}, \mathscr{A}^{1} \psi_{j}\right\rangle}{\left\langle\psi_{j}, \psi_{j}\right\rangle}+O(1 / N) .
$$


Notice that $\psi_{j}$ and the integral kernel $\mathscr{A}^{1}\left(z, z^{\prime}\right)$ are smooth and rapidly decaying so that the error in replacing the discrete scalar product $(\cdot, \cdot)$ by the continuum $\langle\cdot, \cdot\rangle$ is $O\left(1 / N^{k}\right)$ with $k$ arbitrarily large.

From (5.47)

$$
\begin{aligned}
\gamma_{j} & =\left(\Sigma \psi_{j}, A \Sigma \psi_{j}\right) \\
& =\left\langle\psi_{j}, \mathscr{A}^{1} \psi_{j}\right\rangle+O(1 / N) \\
& =\mu_{j}^{(0)}+\frac{1}{\sqrt{N}}\left\langle\psi_{j}, \mathscr{V}_{1} \psi_{j}\right\rangle+O(1 / N) .
\end{aligned}
$$

Proceeding as in (5.47) and using

$$
\left\langle\mathscr{A} \psi_{j}, \mathscr{V}_{1} \psi_{j}\right\rangle-\left\langle\psi_{j}, \mathscr{A} \psi_{j}\right\rangle\left\langle\psi_{j}, \mathscr{V}_{1} \psi_{j}\right\rangle=0
$$

we have

$$
\begin{aligned}
\eta_{j} & =\left(\varphi_{j},\left[A-\gamma_{j}\right]^{2} \varphi_{j}\right) \\
& =N^{-1}\left[\left\langle\psi_{j},\left(\mathscr{V}_{1}\right)^{2} \psi_{j}\right\rangle-\left\langle\psi_{j}, \mathscr{V}_{1} \psi_{j}\right\rangle^{2}\right]+O(1 / N) \\
& =O(1 / N) .
\end{aligned}
$$

By Temple, we therefore have

$$
\mu_{j}=\mu_{j}^{(0)}+\frac{1}{\sqrt{N}}\left\langle\psi_{j}, \mathscr{V}_{1} \psi_{j}\right\rangle+O(1 / N)
$$

By parity of the integrand, one finds

$$
\mu_{j}^{(1)}=\left\langle\psi_{j}, \mathscr{V}_{1} \psi_{j}\right\rangle=0
$$

The above procedure can be extended to all orders in $1 / \sqrt{N}$. Quite generally, a parity argument shows that only even powers of $1 / \sqrt{N}$ occur in the expansion for eigenvalues.

\section{Proof of Theorem 1.5}

From (3.22), the fermion two-point function can be written as

where

$$
\left\langle\bar{\psi}_{x} \cdot \psi_{y}\right\rangle_{L}=2 N \frac{\tilde{Z}_{L}^{k l}}{Z_{L}}, \quad k=x / a, l=y / a,
$$

$$
\begin{aligned}
\tilde{Z}_{L}^{k l} & =\int d \mu_{L, 0}(\phi) \operatorname{det}\left(\Gamma_{L}+(\zeta / \sqrt{N}) \Phi_{L}\right)^{2 N}\left(\Gamma_{L}+(\zeta / \sqrt{N}) \Phi_{L}\right)_{k l}^{-1} \\
& =\int d \mu_{L, 0}(\phi) \operatorname{det}\left(\Gamma_{L}+(\zeta / \sqrt{N}) \Phi_{L}\right)^{2 N-1} \operatorname{det}\left(\Gamma_{L}+(\zeta / \sqrt{N}) \Phi_{L}\right)^{(k l)}
\end{aligned}
$$

with $\operatorname{det} A^{(k l)}$ meaning the determinant of the cofactor matrix $A^{(k l)}$ obtained by deleting the $k^{\text {th }}$ row and $l^{\text {th }}$ column of $A$. Let us suppose $k \leqq l$ : the opposite case can be treated in the same way. 
In the free theory $(\zeta=0)$, the two-point function $(6.1)$ is given by $2 N\left(\Gamma_{L}\right)_{k l}^{-1}$. We notice that $\Gamma_{L}$ with $L$ even is invertible and its inverse can be explicitly computed

$$
\Gamma_{L}^{-1}=\left(\begin{array}{cccc}
A & -B^{t} & \ldots & -B^{t} \\
B & A & \ldots & -B^{t} \\
\vdots & \vdots & \ddots & \vdots \\
B & B & \ldots & A
\end{array}\right)
$$

where

$$
A=\left(\begin{array}{cc}
0 & -1 \\
1 & 0
\end{array}\right) \quad \text { and } B=\left(\begin{array}{ll}
0 & 0 \\
1 & 0
\end{array}\right) .
$$

It follows that the free two-point function does not decay as $|x-y| \rightarrow \infty$. On the other hand, Theorem 1.5 states that the two-point function decays exponentially fast for any $\zeta \neq 0$ and $N$ sufficiently large.

Before replacing the determinants in (6.2) by the expansion (2.4) we notice that

$$
\operatorname{det}\left(\Gamma_{L}+\Psi_{L}\right)^{(k l)}=\operatorname{det}\left(\Gamma_{L}+\Psi_{L}\right)^{<k} \operatorname{det}\left(\Gamma_{L}+\Psi_{L}\right)^{>l},
$$

where $A^{<k}$ mean the $(k-1) \times(k-1)$ matrix formed by removing in $A$ all rows and columns whose labels run from $k$ to $L$. Similarly, $A^{>l}$ is a $(L-l-1) \times(L-l-1)$ matrix with the rows and columns from 1 to $l$ removed.

Equation (6.2) can thus be written as

$$
\tilde{Z}_{L}^{k l}=\sum_{\mathbf{X}} \int d \mu_{L, 0}(\phi) \prod_{i=1}^{2 N}\left\{\prod_{y \in X_{i}^{c}}(\zeta / \sqrt{N}) \phi_{y}\right\},
$$

where the summation is now over all collections $\mathbf{X}=\left(X_{1}, \ldots, X_{2 N}\right)$ of compatible sets with $X_{1}$ constrained to have the sites from $k$ to $l$ (ends included) covered by dimers. We notice that this constraint leads $\tilde{Z}_{L}^{k l}$ to vanish for all $k, l$ such that $|k-l|$ is even.

Integrating out the $\phi$-variables and performing a resummation, (6.2) can be written as

$$
\tilde{Z}_{L}^{k l}=(-1)^{k+l} \alpha^{L} \operatorname{Tr}\left(P_{0} T^{k-1} \tilde{S}\left(\tilde{T} \tilde{T}^{t}\right)^{(l-k-1) / 2} \tilde{S} T^{L-l}\right),
$$

where $\tilde{T}$ is the $(N+1) \times(N+1)$ matrix whose entries are given by

$$
\tilde{T}\left(n, n^{\prime}\right)=T\left(n, n^{\prime}\right) \tilde{\Delta}\left(n, n^{\prime}\right)
$$

with $T$ as in $(4.5), \tilde{\Delta}(N x, N y)=\tilde{\delta}(x, y)$ with

$$
\tilde{\delta}(x, y)=\left(\frac{x}{1-y}\right)^{1 / 2}
$$

and $\tilde{S}$ is a matrix which will not be specified since it does not contribute to the mass gap expression.

Repeating the procedure of Sect. 4 , we are able to write the eigenvalues $\left\{\tilde{\lambda}_{j}\right\}_{j=0}^{N}$ of $\tilde{T}$ in the following form:

$$
\tilde{\lambda}_{j}=c_{0} e^{N \bar{f}}\left(\tilde{\mu}_{j}^{(0)}+O\left(\frac{1}{\sqrt{N}}\right)\right), \quad j=0,1, \ldots, n_{0} .
$$


The ratio between the partition function (6.1) can thus be written as

$$
\frac{\tilde{Z}_{L}^{k l}}{Z_{L}}=(-1)^{k+l}\left\langle\tilde{\psi}_{0}|\tilde{S}| \psi_{0}\right\rangle^{2}\left(\frac{\tilde{\lambda}_{0}}{\lambda_{0}}\right)^{|k-l|}+O\left(\left(\lambda_{1} / \lambda_{0}\right)^{L}\right)
$$

which implies a mass gap $\hat{m}$ given by

$$
\hat{m}=\frac{1}{a} \ln \frac{\lambda_{0}}{\tilde{\lambda}_{0}}=\frac{1}{a} \ln \frac{\lambda_{0}^{(0)}}{\tilde{\lambda}_{0}^{(0)}}+O(1 / \sqrt{N}) .
$$

In order to prove Theorem 1.5 we need compute only the leading term in (6.7) and show that it coincides with the mean field mass gap.

Following the procedure in Sect. 4, the matrix $\tilde{A}=c_{0}^{-1} \exp [-N \bar{f}] \tilde{T}$ in the eigenvalue equation is approximated by an integral operator given by

$$
\tilde{\mathscr{A}}=\tilde{\delta}^{\prime} \mathscr{A}+O\left(1 / N^{1 / 2}\right),
$$

where $\mathscr{A}$ is given by $(4.16)$ and

$$
\tilde{\delta}^{\prime}=\tilde{\delta}(\bar{x}, \bar{x})=\left(\frac{\bar{x}}{1-\bar{x}}\right)^{1 / 2}
$$

In view of (6.7), Lemma 4.1 and (1.8),

$$
\begin{aligned}
\hat{m} & =\frac{1}{a} \ln \frac{1}{\tilde{\delta}^{\prime}}+O(1 / \sqrt{N}) \\
& =\frac{1}{2 a} \ln \left[\zeta^{2}+\left(1+\zeta^{4}\right)^{1 / 2}\right]+O(1 \sqrt{N}),
\end{aligned}
$$

which by comparing to the mean field mass gap $\hat{m}_{M F}$ in (1.5), concludes the proof of Theorem 1.5.

\section{Concluding Remarks}

In the preceding section we have shown that the fermion correlator exhibits an exponential decay rate which is close to the mean field value $\hat{m}_{M F}$. Another important aspect of the model is the "bosonic" correlator

$$
\left\langle[\bar{\psi} \cdot \psi]_{x}[\bar{\psi} \cdot \psi]_{y}\right\rangle_{L}=\frac{N}{\zeta^{2}} \int d \mu(\phi) \phi_{i} \phi_{j}
$$

Let us begin by defining

where

$$
\int d \mu_{L}(\phi) \phi_{i} \phi_{j} \equiv \frac{N}{\zeta^{2}} \frac{Z_{L}^{i j}}{Z_{L}},
$$

$$
Z_{L}^{i j}=\int d \mu_{L, 0}(\phi) \operatorname{det}\left(\Gamma_{L}+(\zeta / \sqrt{N}) \phi\right)(\zeta / \sqrt{N}) \phi_{i}(\zeta / \sqrt{N}) \phi_{j} ; \quad i<j \in \mathscr{L}
$$

is a modified partition function. 
Following the procedure in Sect. 4, (7.2) can be written as

$$
Z_{L}^{i j}=\sum_{\mathbf{X}} \int d \mu_{L, 0}(\phi) \prod_{i=1}^{2 N}\left[\prod_{z \in X_{l}^{c}}(\zeta / \sqrt{N}) \phi_{z}\right](\zeta / \sqrt{N}) \phi_{i}(\zeta / \sqrt{N}) \phi_{j}
$$

with $\mathbf{X}=\left(X_{1}, \ldots, X_{2 N}\right)$ running, as before, over all collections of $2 N$ compatible sets.

Performing the Gaussian integral and some combinatorics, (7.3) can be written as

$$
Z_{L}^{i j}=\alpha^{L} \operatorname{Tr}\left(P_{0} T_{e}^{i} \bar{S} T_{o}^{j-i+1} \bar{S} T_{e}^{L-j-1}\right),
$$

where $T_{e} \equiv T$ ("e" for "even") is given by (4.5), and

$$
T_{o}\left(n, n^{\prime}\right)=T_{e}\left(n+1 / 2, n^{\prime}+1 / 2\right) .
$$

The important point is that the spectrum of the matrix $T_{o}$ equals that of the matrix $T_{e}$, to all orders in the $1 / N$ expansion. This is simply because the continuum approximation of $T_{o}$ is precisely that of $T_{e}$, with the arguments $z, z^{\prime}$ shifted by $1 / \sqrt{N}$. In particular,

$$
\lambda_{0, o}=\lambda_{0, e}+O\left(1 / N^{k}\right)
$$

for any $k$. Since an exponential decay rate for the bosonic correlator is a consequence of a gap between these two eigenvalues, in principle, the $1 / N$ expansion is incapable of detecting such a decay rate.

For $N$ finite, it is thus uncertain whether $\lambda_{0, o}<\lambda_{0, e}$. Numerical computations so far indicate that while this inequality is true for most $N, \zeta$, it can be violated in extreme cases.

To answer why the fermion correlator has an $O(1)$ gap, we note that the model has two "superselection" sectors, odd and even. The partition function $Z$ given by (4.4) is in fact restricted to the even sector because of the choice of boundary conditions, and furthermore, the fermion field operator maps even to even and odd to odd. The bosonic operator, however, maps even to odd and vice versa.

A detailed investigation of these questions is the subject of a paper in preparation [FHM].

Acknowledgements. P.A.F. da Veiga would like to thank Prof. A. Jaffe for support and hospitality at Harvard University in the beginning of this project. D.H.U. Marchetti thanks G. Slade and T. Hurd for their hospitality at McMaster University.

\section{References}

[BG] Balaban, T., Gawedzki, K.: A low temperature expansion for the pseudoscalar Yukawa model of quantum fields in two space-time dimensions. Ann. 1'Inst. H. Poincaré 36, 4, 272 (1982)

[CE] Collet, P., Eckmann, J.P.: A renormalization group analysis of the hierarchical model in statistical mechanics. Lect. Notes in Phys. 74 (1978)

[FHM] Faria da Veiga, P.A., Hurd, T.R., Marchetti D.H.U.: Work in preparation

[FMRS] Feldman, J., Magnen, J., Rivasseau, V., Sénéor, R.: A renormalizable field theory: The massive Gross-Neveu model in two dimensions. Commun. Math. Phys. 103, 67 (1986)

[G] Griffiths, R.B.: Peierls' proof of spontaneous magnetization in a two-dimension Ising ferromagnet. Phys Rev. A136, 437 (1964) 
[GJS] Glimm, J., Jaffe, A., Spencer, T.: A convergent expansion about mean field theory: 1. The expansion. Ann. Phys. 101, 610 (1976)

[GK] Gawedzki, K., Kupiainen, A.: Gross-Neveu model through convergent perturbation expansion. Commun. Math. Phys. 102, 1 (1985)

[GN] Gross, D., Neveu, A.: Dynamical symmetry breaking in asymptotically free field theories. Phys. Rev. D10, 3235 (1974)

[KMR] Kopper, C., Magnen, J., Rivasseau, V.: Mass generation in the large $N$ Gross-Neveumodel. Commun. Math. Phys. 169, 121-179 (1995)

[L] Lieb, E.H.: Models in Statistical Mechanics. Lectures in Theoretical Physics, New York: Gordon and Breach, 1969

[NJ] Nambu, Y., Jona-Lasinio, G.: Dynamical model of elementary particles based on an analogy with superconductivity: I. Phys. Rev. 122, 345 (1961)

[RS] Reed, M., Simon, B.: Methods of Modern Mathematical Physics IV: Analysis of operators. New York: Academic Press, 1978

Communicated by D. Brydges 